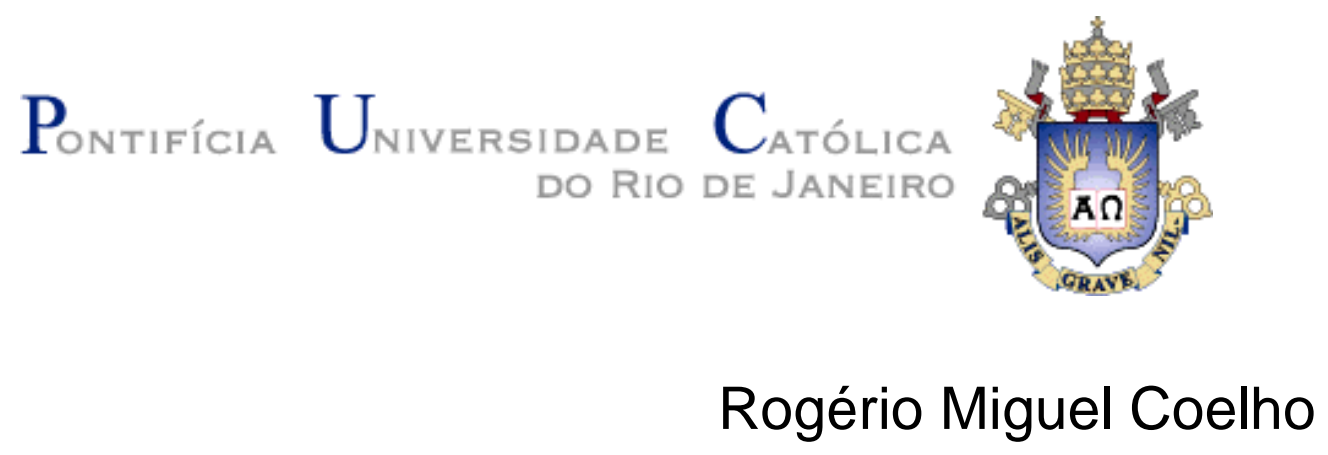

\author{
Integração de Ferramentas \\ Gráficas e Declarativas na Autoria \\ de Arquiteturas Modeladas através \\ de Grafos Compostos
}

DissertaÇÃo de Mestrado

DEPARTAMENTO DE INFORMÁTICA

Programa de Pós-Graduação em Informática

Rio de Janeiro

Agosto de 2004 


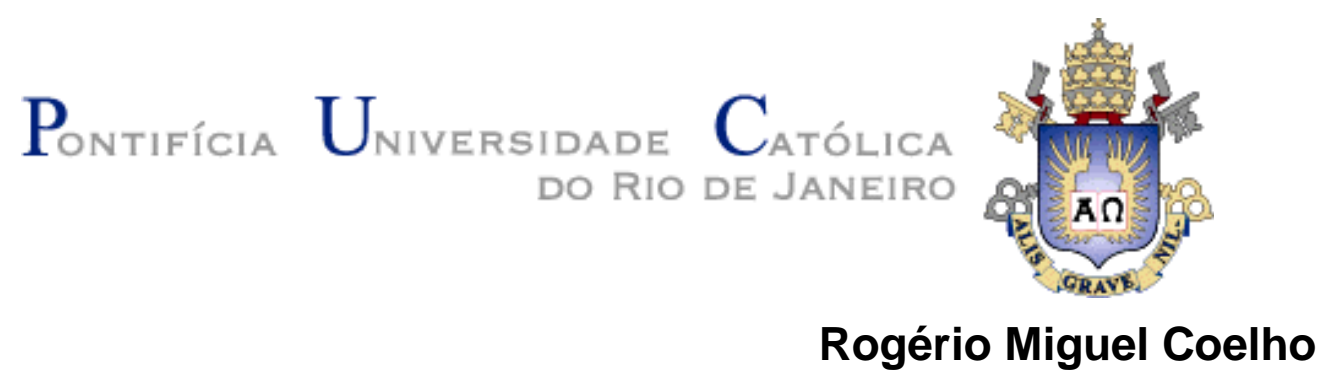

Integração de Ferramentas Gráficas e Declarativas na Autoria de Arquiteturas Modeladas através de Grafos Compostos

Dissertação apresentada como requisito parcial para obtenção do título de Mestre pelo Programa de PósGraduação em Informática da PUC-Rio.

Orientador: Luiz Fernando Gomes Soares Co-orientador: Rogério Ferreira Rodrigues

Rio de Janeiro, agosto de 2004 


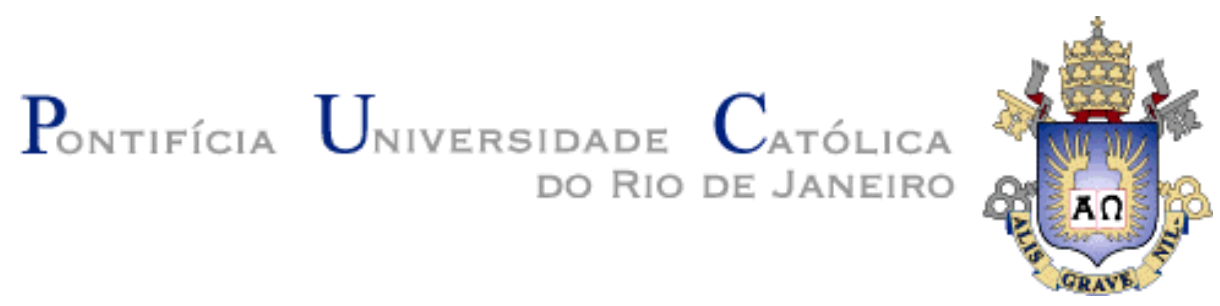

Rogério Miguel Coelho

\title{
Integração de Ferramentas Gráficas e Declarativas na Autoria de Arquiteturas Modeladas através de Grafos Compostos
}

\begin{abstract}
Dissertação apresentada como requisito parcial para obtenção do título de Mestre pelo Programa de PósGraduação em Informática da PUC-Rio. Aprovada pela Comissão Examinadora abaixo assinada.
\end{abstract}

\author{
Luiz Fernando Gomes Soares \\ Orientador \\ Departamento de Informática - PUC-Rio \\ Rogério Ferreira Rodrigues \\ Co-orientador \\ Departamento de Informática - PUC-Rio \\ Simone Diniz Junqueira Barbosa \\ Departamento de Informática - PUC-Rio \\ Sergio Colcher \\ Departamento de Informática - PUC-Rio \\ José Eugênio Leal
} Coordenador(a) Setorial do Centro Técnico Científico - PUC-Rio 
Todos os direitos reservados. É proibida a reprodução total ou parcial do trabalho sem autorização da universidade, do autor e do orientador.

\section{Rogério Miguel Coelho}

Graduado em Engenharia de Computação pela Universidade Federal do Espírito Santo (UFES) em 2001. Atualmente, integra o grupo de pesquisadores do Laboratório TeleMídia da PUC-Rio, desenvolvendo pesquisa na área de Sistemas Hipermídia.

Ficha Catalográfica

Coelho, Rogério Miguel
Integração de ferramentas gráficas e
declarativas na autoria de arquiteturas modeladas através
de grafos compostos / Rogério Miguel Coelho ;
orientador: Luiz Fernando Gomes Soares ; co-orientador:
Rogério Ferreira Rodrigues. - Rio de Janeiro : PUC-Rio,
Departamento de Informática, 2004.
109 f. : il. ; 30 cm
Dissertação (mestrado) - Pontifícia Universidade
Católica do Rio de Janeiro, Departamento de Informática.
Inclui referências bibliográficas
1. Informática - Teses. 2. Autoria de
Ferquiteturas. 3. Grafos compostos. 4. Olho-de-peixe. I.
Soares, Luiz Fernando Gomes. II. Rodrigues, Rogério
Janeiro. Departamento de Informática. III. Título.

CDD: 004 
Este trabalho é dedicado: A meus pais Louise, Ary, Manuel e a minha irmã Lorena. A toda amada família Miguel (Queridos avós, tios e primos). A Deus pela dádiva por poder contar com tantas pessoas maravilhosas em minha vida. 


\section{Agradecimentos}

Ao meu orientador, Prof. Luiz Fernando, por acreditar em meu potencial e por todo seu apoio nos momentos alegres e difíceis: "Capoeira que é bom não cai, mas seu um dia ele cai, cai bem".

Ao meu co-orientador, Prof. Rogério Rodrigues, por sua atenção, paciência e boa vontade em me ensinar. Tendo sido um verdadeiro guia para o desenvolvimento deste trabalho, sua ajuda foi fundamental.

Aos meus colegas do TeleMídia, pelo companheirismo e ajuda prestados. Em especial a Heron Vilela (Filhão) pela amizade e ajuda no desenvolvimento do "V0" e, a Sergio Cavendish pela revisão deste trabalho.

Agradecimentos especiais à minha família: Louise (minha mãe), Ary (meu pai, in memorian), Manuel e Lô pelo amor, carinho e incentivo. Ao meu irmão mais velho, Flavio Varejão, pelos conselhos e ombro amigo.

À Reivani, pelo amor, companheirismo e por todos os momentos que passamos juntos aqui no Rio de Janeiro.

Aos Professores da Universidade Federal do Espírito Santo por minha formação na Graduação, sem a qual não teria chegado até aqui.

À CAPES e ao FUNTTEL pelo apoio financeiro.

E a todos aqueles que direta ou indiretamente contribuíram para realização deste trabalho. 


\section{Resumo}

Coelho, Rogério Miguel. Integração de Ferramentas Gráficas e Declarativas na Autoria de Arquiteturas Modeladas através de Grafos Compostos. Rio de Janeiro, 2004. 108p. Dissertação de Mestrado Departamento de Informática, Pontifícia Universidade Católica do Rio de Janeiro.

Este trabalho descreve um conjunto de ferramentas para auxiliar na construção de aplicativos para autoria de arquiteturas de sistemas baseadas em grafos compostos. As ferramentas são divididas em quatro visões de grafos compostos: uma visão gráfica estrutural, uma visão gráfica temporal, uma visão gráfica espacial e uma visão textual. As quatro visões funcionam de maneira sincronizada, a fim de oferecer um ambiente integrado de autoria. As visões são providas de mecanismos de filtragem para auxiliar na especificação de arquiteturas mais complexas. As ferramentas desenvolvidas foram diretamente aplicadas ao domínio de autoria de documentos hipermídia, mas podem ser utilizadas em outros domínios, como na definição de arquiteturas de sistemas de software, ferramentas de especificação formal, projeto de workflows, entre outros.

\section{Palavras-chave}

Autoria de Arquiteturas; Grafos Compostos; Olho-de-Peixe; Sistemas Hipermídia 


\section{Abstract}

Coelho, Rogério Miguel. Integration of Graphical and Declarative Tools for Authoring System Architectures Based on Composite Graphs. Rio de Janeiro, 2004. 108p. Master Thesis - Departamento de Informática, Pontifícia Universidade Católica do Rio de Janeiro.

This work presents a set of tools to help the implementation of applications for authoring system architectures based on composite graphs. The tools are divided in four graph views: structural, temporal, spatial, and declarative. The views are synchronized in order to offer an integrated authoring environment. Moreover, the views include filtering mechanisms to simplify the specification of more complex architectures. The tools were specialized to hypermedia authoring, but they can be used in other domains, such as software architecture specifications, formal specification tools, workflow projects, etc.

\section{Key words}

Authoring System Architectures; Composite Graph; Fish-Eye; Hypermedia Systems 


\section{Sumário}

1 Introdução 13

1.1. Motivação 13

1.2. Objetivos 20

$\begin{array}{ll}\text { 1.3. Estrutura da Tese } & 21\end{array}$

2 Conceitos Preliminares $\quad 22$

2.1. Definição de Grafos Compostos 22

2.2. Sistemas Modelados através de Grafos Compostos 24

2.2.1. ADLs (Architecture Description Languages) 24

2.2.2. Forma 30

2.2.3. Workflow 31

2.3. Modelos Hipermídia 34

2.3.1. Modelo Conceitual NCM (Nested Context Model) 36

2.3.2. Linguagem NCL (Nested Context Language) 38

3 Ferramentas para Edição de Arquiteturas de Sistemas Baseadas em Grafos Compostos

3.1. Edição Textual 41

3.2. Edição Gráfica 44

3.2.1. Visão Gráfica Estrutural $\quad 45$

3.2.2. Visão Gráfica Temporal 49

3.2.3. Visão Gráfica Espacial 51

3.3. Sincronização entre as visões 53

4 Técnicas de Filtragens Aplicadas às Visões do Ambiente de Autoria do $\begin{array}{ll}\text { Sistema HyperProp } & 58\end{array}$

4.1. Técnica de Filtragem Olho-de-Peixe aplicada em Grafos Compostos58

4.2. Filtragem Olho-de-Peixe no Sistema HyperProp 63

4.2.1. Filtragem olho-de-peixe na Visão Estrutural 63 
4.2.2. Visão olho-de-peixe na Visão Espacial e Textual

4.3. Cálculos Realizados na Filtragem Olho-de-Peixe sobre a Visão Espacial

5 Trabalhos Relacionados

74

5.1. GraphViz

74

5.1.1. Construtor dot

76

5.1.2. Construtor neato

77

5.1.3. Construtor twopi

78

5.1.4. Comparações entre o GraphViz e HyperProp

80

5.2. SALIX

81

5.2.1. Comparações entre o SALIX e HyperProp 83

5.3. Kaomi

84

5.3.1. Comparações entre o Kaomi e HyperProp 86

5.4. GRiNS

86

5.4.1. Visão de Apresentação

87

5.4.2. Visão Temporal

88

5.4.3. Visão Espacial

89

5.4.4. Visão Declarativa

90

5.4.5. Comparações entre o Sistema GRiNS e o HyperProp

91

5.5. Outras Ferramentas de Edição

92

5.5.1. Ariadne

92

5.5.2. Arakne

94

6 Conclusões

98

6.1. Trabalhos Futuros

99

7 Referências Bibliográficas

101

8 Apêndice 1 GXL (Graph eXchange Language)

106 


\section{Lista de figuras}

$\begin{array}{ll}\text { Figura 1-1 Subsistemas de um sistema hipermídia } & 14\end{array}$

$\begin{array}{ll}\text { Figura 1-2 Visões de Documentos Hipermídia } & 17\end{array}$

Figura 2-1 Grafos Compostos com Composicionalidade 24

Figura 2-2 Representação de uma arquitetura de software descrita através de uma

ADL utilizando grafos compostos 26

Figura 2-3 Documento HTML 27

Figura 2-4 Documento xADL 29

Figura 2-5 Arquitetura de Forma em Grafos Compostos 31

Figura 2-6 Workflow mapeado em grafos compostos 32

Figura 2-7 Um exemplo de Workflow em XRL 34

Figura 2-8 Modelo NCM representado em Grafos Compostos 35

Figura 2-9 Elo Multiponto em Grafos Compostos 36

Figura 2-10 Exemplo de documento NCL 2.0 39

Figura 3-1 Visão declarativa do sistema HyperProp 42

Figura 3-2 Validação de documentos XML 43

Figura 3-3 Editor declarativo com documento GXL 44

Figura 3-4 Visão estrutural 46

Figura 3-5 Visão Temporal $\quad 50$

Figura 3-6 Visão Espacial para edição de Layout de apresentação. 52

Figura 3-7 - Arquitetura de integração das visões $\quad 54$

Figura 3-8 Visão Espacial sincronizada com Visão Textual 56

Figura 4-1 Exemplo de cálculo de API(x) para grafos compostos 60

Figura 4-2 Exemplo de cálculo de $D c(x, y)$ para grafos compostos 61

Figura 4-3 Exemplo de cálculo de $D e(x, y)$ para grafos compostos 62

Figura 4-4 Exemplo de cálculo da função $D(x, y)$ para grafos compostos baseado nas Figuras 4-2 $(D c(x, y))$ e 4-3 $(D e(x, y)) \quad 62$

Figura 4-5 Exemplo de cálculo da função $\operatorname{DOI}(x, y)$ para grafos compostos baseado nas Figuras 4-1 (API $(x))$ e 4-4 $(D(x, y)) \quad 63$

Figura 4-6 Visão estrutural sem a técnica olho-de-peixe 64

Figura 4-7 Visão olho-de-peixe com 25\% de Nível de detalhe 65 
Figura 4-8 Visão declarativa sincronizada com visão estrutural (olho-de-peixe $25 \%)$

Figura 4-9 Visão olho-de-peixe com 75\% de Nível de detalhe

Figura 4-10 Visão declarativa sincronizada com visão estrutural (olho-de-peixe $75 \%)$

Figura 4-11 Visão Espacial da especificação do elemento layout na linguagem NCL

Figura 4-12 Visão Textual da especificação do elemento layout refletida da Figura 4-11

Figura 4-13 Visão olho-de-peixe aplicada na visão espacial

Figura 4-14 Visão olho-de-peixe aplicada na visão textual

Figura 4-15 Cálculo da função API(x,y)

Figura 4-16 Cálculo da função D(x,y)

Figura 4-17 Cálculo da função DOI(x,y)

Figura 5-1 Sistema GraphViz 75

Figura 5-2 Grafo Hierárquico (dot) 76

Figura 5-3 Grafo não direcionado (neato) 78

Figura 5-4 Grafo circular simples (twopi) 79

Figura 5-5 Grafo circular com sobreposição (twopi) 79

Figura 5-6 Grafo circular sem sobreposição (twopi) 80

Figura 5-7 Ambiente SALIX (Autoria Declarativa) 82

Figura 5-8 Ambiente SALIX (Autoria em árvore e tabelas) 83

Figura 5-9 Janela principal do ambiente GRiNS

Figura 5-10 Visão de apresentação do sistema GRiNS 88

Figura 5-11 Visão Temporal do GRiNS $\quad 89$

Figura 5-12 Visão Espacial do sistema GRiNS 90

Figura 5-13 Visão Textual do GRiNS 91

Figura 5-14 Mapa de navegação da ferramenta Ariadne 94

Figura 5-15 Ambiente Arakne 96

Figura 8-1 Exemplo simples de GXL 106

$\begin{array}{ll}\text { Figura 8-2 Grafo GXL com elemento rel } & 107\end{array}$

$\begin{array}{ll}\text { Figura 8-3 Grafos compostos em GXL } & 108\end{array}$ 